\title{
Exploiting the in-situ Electrical X-ray Microscopy for Semiconductor Nano Devices Analysis by X-ray Nanoprobe Beamline at Taiwan Photon Source.
}

\author{
Shao-Chin Tseng ${ }^{1, *}$, Bi-Hsuan Lin ${ }^{1}$, Xiao-Yun Li ${ }^{1}$, Yu-Sheng Lai ${ }^{2}$, Po-Hsien Tseng ${ }^{3}$, Chien-Yu Lee ${ }^{1}$, \\ Bo-Yi Chen ${ }^{1}$, Gung-Chian Yin ${ }^{1}$, Ming-Ying Hsu ${ }^{1}$, Shih-Hung Chang ${ }^{1}$ and Mau-Tsu Tang ${ }^{1}$ \\ 1. National Synchrotron Radiation Research Center, Hsinchu 30076, Taiwan. \\ 2. National Nano Device Laboratories, Hsinchu 30078, Taiwan. \\ 3. Department of Materials Science and Engineering, National Chiao Tung University, Hsinchu 30010, \\ Taiwan. \\ * Corresponding author, email_tseng.sc@nsrrc.org.tw
}

The development of X-ray microscopy for fundamental scientific and industrial technology analysis is very important for the progress in scientific research. The complete and integrated facilities and tools can help users to get more insight into the basic property of the science research and the underlying physical mechanism. The X-ray nanoprobe (XNP) provides versatile X-ray-based inspection technologies, including diffraction, absorption spectroscopy, imageology, and so on. The XNP beamline particularity is listed in the followings: 1 .Energy range: $4-15 \mathrm{kev} 2$.Photon flux: $\geqq 10^{10}$ photons/sec 3.Energy resolution $\Delta \mathrm{E} / \mathrm{E}: \leqq 2 * 10^{-4}$ with $\mathrm{Si}(111)$ crystal 4 . Beam size at focal point: $\leqq 50 \mathrm{~nm} * 50 \mathrm{~nm}$ at $10 \mathrm{kev}\left(\mathrm{H}^{*} \mathrm{~V}, \mathrm{FWHM}\right) 5$.High-order harmonics: $\leqq 1 * 10^{-3} 6$.Working distance (from the end of focusing optics to the focal point): $\geqq 20 \mathrm{~mm}$. Also, it will improve the analysis scale of inhomogeneous materials, tiny and diluted samples to the nanoscale. The primary experimental technique of XNP includes X-ray fluorescence spectroscopy (for the analysis of the 3D distribution of elements), extended X-ray absorption spectroscopy (for the analysis of the electronic configuration at the atomic or molecular bonding length), X-ray excited optical luminescence [1-2] (for the analysis of the recombination and transport of carriers), in-phase scanning X-ray imaging (the Fourier phase transform calculation can improve the spatial resolution down to $3 \mathrm{~nm}$ to $5 \mathrm{~nm}$, and detect the stress distribution inside the nanostructures)[3]. Figure 1 shows the XNP beamline endstation. Moreover, the high-transmitted XNP can be used to inspect the "Nano World" like atomic arrangements, chemical and electronic configurations, which are widely adopted in the physics, chemistry, materials science, semiconductor devices, nanotechnologies.

The semiconductor industrial technology makes the world faster and more convenient. However the semiconductor device scale down to nano size also has some of the problems especially electromigration. The electromigration effect will make the device invalid after a period of operation time. This will greatly reduce the stability of semiconductor nano device. Figure 2 a shows the mechanism of electromigration. This effect involves metal mass transfer and creates voids in metal connection layers. These voids increases the resistance of the metal connections leading to device failure. Figure $2 b$ shows the silicon drift detector. Figure $2 \mathrm{c}$ shows ion chamber system. The electromigration effect at nano channel is analyszed by XAS measured using the ion chamber and silicon drift detector. This device structure was very suitable for observing the electromigration effect in cooperation with National Nano Device Laboratories.

In this study [4], we combined the 13 electrical contact sample stage to develop the in-situ electrical X-ray microscopy. The 13 electrical contacts sample stage was produced by Ferrovac GmbH. Figure 3 shows the in-situ electrical sample stage installed in the XNP chamber. The Keithley 2450 is a source 
meter for electrical measurement and power supplying. The in-situ electrical sample stage was connected via feedthrough. The in-situ electrical XRF imaging observes metal atoms migration phenomenon when the device is in operation. That revelas the electromigration mechanism.

\section{Reference:}

[1] Bi-Hsuan Lin, Huang-Yeh Chen, Shao-Chin Tseng, Jian-Xing Wu, Bo-Yi Chen, Chien-Yu Lee, Gung-Chian Yin, Shih-Hung Chang, Mau-Tsu Tang, and Wen-Feng Hsieh, Applied Physics Letters 109, 192104 (2016).

[2] Bi-Hsuan Lin, Yung-Chi Wu, Huang-Yeh Chen, Shao-Chin Tseng, Jian-Xing Wu, Xiao-Yun Li, BoYi Chen, Chien-Yu Lee, Gung-Chian Yin, and Shih-Hung Chang, Optics Express 26, 2731-2739 (2018).

[3] Martin V. Holt, Stephan O. Hruszkewycz, Conal E. Murray, Judson R. Holt, Deborah M. Paskiewicz,2 and Paul H. Fuoss, Physical Review Letters 112, 165502 (2014)

[4] Ministry of Science and Technology of Taiwan (105-2112-M-213-011-MY2) and the National Synchrotron Radiation Research Center provided support for this research.

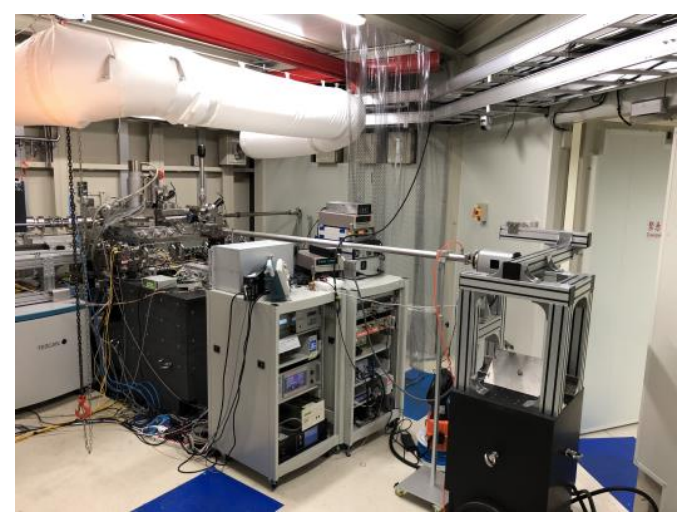

(a)

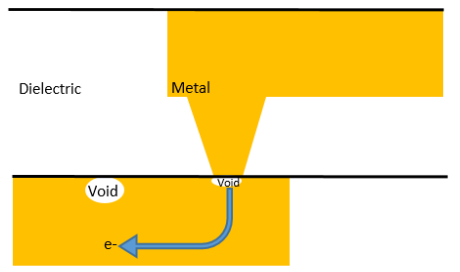

Figure. 1. The X-ray nanoprobe beamline endstation in hutch.

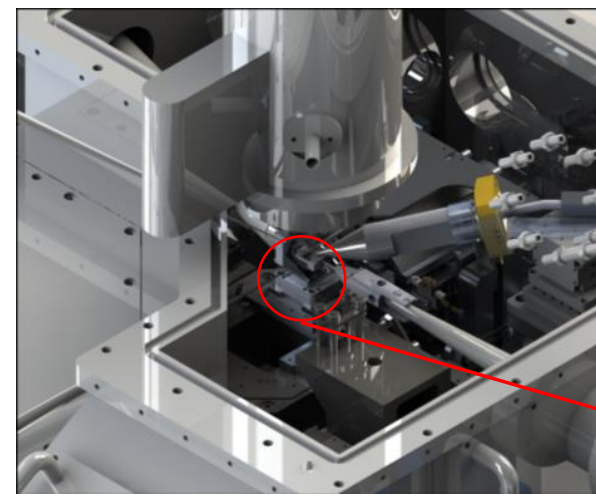

Figure. 3. Schematic diagram of in-situ electrical sample stage.
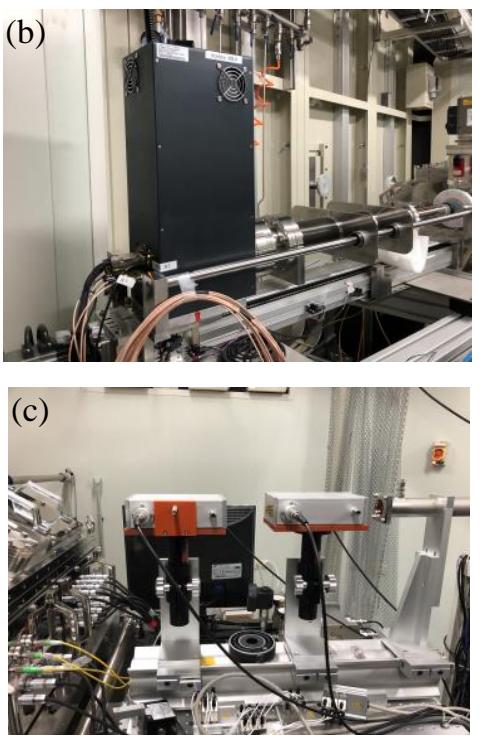

Figure. 2. (a) Schematic diagram of electromigration (b) Silicon drift detector (c) Ion chamber

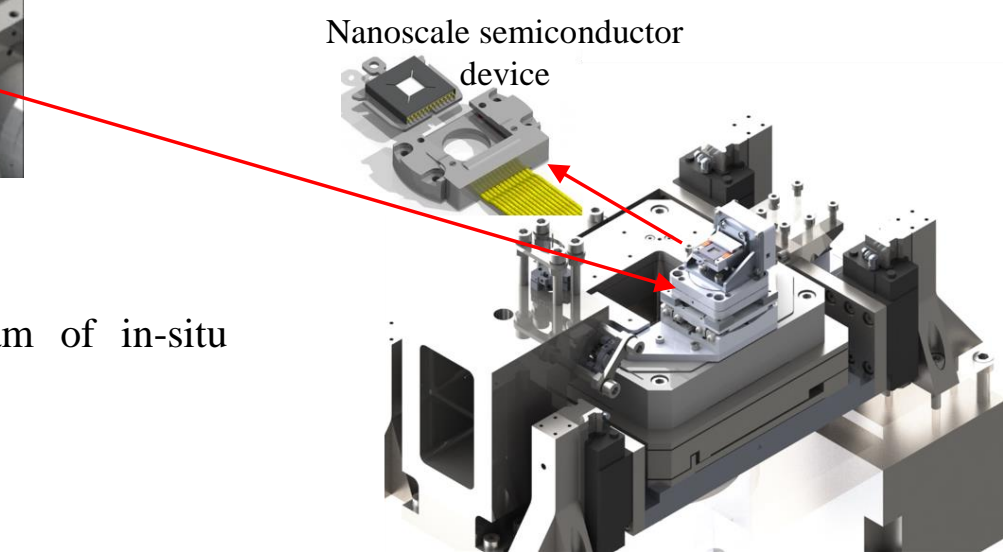

